\title{
Transparencia: compromisos en el marco de las elecciones 2005 en Chile
}

\begin{abstract}
A continuación presentamos unos cuadros que resumen las respuestas de los candidatos a diputados y senadores a las preguntas formuladas por Chile Transparente en torno a temas de transparencia y anticorrupción, en el marco del proceso eleccionario de renovación de la Cámara de Diputados y Senadores de Chile en diciembre del año 2005. En la sección Nacional de este Anuario está disponible el texto Elecciones 2005. Compromisos de los candidatos presidenciales y parlamentarios en materia de probidad y transparencia de Jacqueline Plass, quien analizó estos resultados y diseñó los cuadros que en esta sección se presentan.

Asimismo, hemos adjuntado las respuestas enviadas por la Presidenta Michelle Bachelet a Transparencia Internacional relativas a la lucha contra la corrupción.
\end{abstract}




\begin{tabular}{|c|c|c|c|c|c|c|c|c|c|c|c|c|c|c|c|c|c|c|c|c|c|c|c|}
\hline \multirow{3}{*}{ } & $\therefore$ & $\begin{array}{l}0 \\
0 \\
0 \\
0 \\
\mathrm{c}\end{array}$ & $\begin{array}{l}\stackrel{0}{0} \\
\text { iे }\end{array}$ & 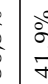 & $\begin{array}{l}0 \\
= \\
\end{array}$ & 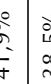 & 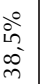 & $\begin{array}{l}\stackrel{0}{\circ} \\
\stackrel{i}{n} \\
m\end{array}$ & $\begin{array}{l}\stackrel{0}{\circ} \\
\text { \&े }\end{array}$ & $\begin{array}{l}\stackrel{0}{0} \\
\stackrel{\circ}{q}\end{array}$ & $\begin{array}{l}0 \\
\text { ○े } \\
\text { in }\end{array}$ & $\begin{array}{l}0 \\
\stackrel{0}{0} \\
\text { m. }\end{array}$ & $\begin{array}{l}\stackrel{े}{2} \\
\text { ले }\end{array}$ & $\begin{array}{l}\stackrel{0}{\circ} \\
\mathrm{o}^{\circ}\end{array}$ & $\begin{array}{l}\stackrel{0}{0} \\
\dot{m}^{2}\end{array}$ & 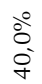 & \begin{tabular}{l}
0 \\
$\stackrel{0}{0}$ \\
\multirow{f}{*}{}
\end{tabular} & $\begin{array}{l}\dot{0} \\
\dot{0} \\
\text { in }\end{array}$ & $\begin{array}{c}\stackrel{0}{0} \\
m^{2}\end{array}$ & $\mid \begin{array}{l}\stackrel{0}{2} \\
\stackrel{y}{\sigma}\end{array}$ & $\frac{\circ}{\stackrel{+}{+}}$ & $\begin{array}{l}\stackrel{\circ}{+} \\
\stackrel{+}{+}\end{array}$ & $\begin{array}{l}\stackrel{0}{\circ} \\
\text { ò }\end{array}$ \\
\hline & $\begin{array}{l}+ \\
+ \\
\dot{5} \\
\underline{\underline{b}}\end{array}$ & $\stackrel{q}{+}$ & ڤֶ) & $\bar{m}$ & $\bar{n} \bar{m}$ & & $\stackrel{m}{=}$ & $\infty$ & $\stackrel{\mathscr{m}}{=}$ & $\stackrel{\infty}{0}$ & q & $\grave{\imath}$ & $\stackrel{\infty}{ \pm}$ & $N$ & 6 & $\stackrel{L n}{\longrightarrow}$ & ลิ & $a$ & $m$ & $\therefore$ & $a$ & $\sigma$ & $\stackrel{R}{f}$ \\
\hline & 峁 & กొ & શิ & 9 & $=9$ & & เn & $m$ & 5 & $\bar{m}$ & ণ & $\stackrel{\circ}{\circ}$ & 吕 & $\stackrel{\infty}{\sim}$ & $\stackrel{\sim}{\sim}$ & 0 & $m$ & in & - & $n$ & + & + & ळ \\
\hline \multirow{3}{*}{ 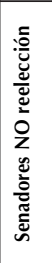 } & ه & iे & $\stackrel{\circ}{0}$ & 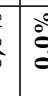 & $\begin{array}{lll}0 \\
:\end{array}$ & & $\begin{array}{l}\stackrel{8}{\circ} \\
8 \\
8 \\
8\end{array}$ & & $\stackrel{\circ}{\circ}$ & & & & $\begin{array}{l}0 \\
\infty \\
1 \\
1\end{array}$ & - & $\begin{array}{l} \\
0\end{array}$ & & $\stackrel{\circ}{0}$ & & & & $\begin{array}{l}0 \\
\stackrel{0}{0}\end{array}$ & & 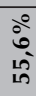 \\
\hline & 絫 & $a$ & - & . & \begin{tabular}{l|l} 
& +
\end{tabular} & & N & & 0 & & & & $a$ & $\sigma$ & Ln & & 0 & & & & 0 & & $\stackrel{\infty}{-}$ \\
\hline & 品 & m & 0 & 0 & $\Rightarrow$ & & N & & 0 & & & & $\wedge$ & + & n & & 0 & & & & 0 & & $\stackrel{0}{\circ}$ \\
\hline \multirow{3}{*}{ 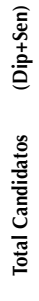 } & ๑ & $\begin{array}{l}\stackrel{0}{1} \\
10 \\
\text { ले }\end{array}$ & $\begin{array}{l}\therefore \\
0 \\
0 \\
\text { in }\end{array}$ & $\left\{\begin{array}{l}\infty \\
\infty \\
7\end{array}\right.$ & $\begin{array}{llll}0 & 0 \\
0 & 0 \\
& 0 & 0 \\
7\end{array}$ & & 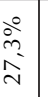 & $\begin{array}{l}\stackrel{\circ}{i n} \\
\stackrel{m}{m}\end{array}$ & $\begin{array}{l}\stackrel{\circ}{0} \\
\text { ڤั) }\end{array}$ & 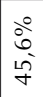 & $\begin{array}{l}\text { 今̊ } \\
\text { in }\end{array}$ & $\begin{array}{l}\stackrel{0}{0} \\
\hat{m}\end{array}$ & $\begin{array}{l}0 \\
0 \\
0 \\
0\end{array}$ & $\begin{array}{l}\stackrel{\circ}{0} \\
\text { L̂ } \\
m\end{array}$ & $\begin{array}{l}\stackrel{\circ}{\circ} \\
\stackrel{n}{n}\end{array}$ & $\begin{array}{l}\AA_{0}^{\circ} \\
\vdots \\
\dot{q}\end{array}$ & \begin{tabular}{l}
$\stackrel{0}{0}$ \\
$\dot{0}$ \\
\multirow{f}{*}{}
\end{tabular} & $\begin{array}{l}\dot{Q}^{\circ} \\
\text { in }\end{array}$ & $\begin{array}{c}\stackrel{0}{m} \\
m_{m}^{2}\end{array}$ & $\mid \begin{array}{l}\stackrel{\circ}{i} \\
\frac{\gamma}{\sigma}\end{array}$ & $\begin{array}{l}\stackrel{\circ}{+} \\
\stackrel{+}{+}\end{array}$ & $\begin{array}{l}\stackrel{\circ}{+} \\
\dot{f}\end{array}$ & $\begin{array}{l}\vdots \\
\text { ¿ें } \\
\text { ले }\end{array}$ \\
\hline & 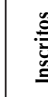 & $\stackrel{f}{I}$ & 능 & 2 & $i 2$ & & $\mp$ & $\infty$ & $\stackrel{\stackrel{L}{m}}{\stackrel{n}{n}}$ & $\stackrel{\infty}{\infty}$ & q & $\hat{\imath}$ & के & 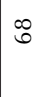 & ட̊ & $\stackrel{\llcorner}{\sim}$ & तิ & $a$ & $m$ & 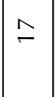 & $a$ & $\sigma$ & ขี \\
\hline & 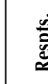 & เก & ิ & 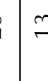 & $=7$ & & $m$ & $m$ & 5 & $\bar{m}$ & $\stackrel{\sim}{\sim}$ & $\because$ & in & $\stackrel{\sim}{\sim}$ & $\bar{\sim}$ & 0 & $m$ & Ln & - & $\wedge$ & $\theta$ & + & 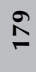 \\
\hline \multirow{3}{*}{ 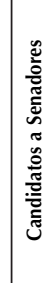 } & $0^{\circ}$ & $\begin{array}{l}\stackrel{0}{0} \\
\stackrel{8}{+9}\end{array}$ & $\begin{array}{l}\stackrel{0}{m} \\
m \\
m\end{array}$ & 8 & 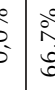 & 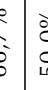 & $\begin{array}{l}0 \\
8 \\
0 \\
\vdots \\
i n\end{array}$ & $\begin{array}{l}\circ \\
\stackrel{0}{ } \\
\stackrel{8}{0}\end{array}$ & $\begin{array}{l}\circ \\
\vdots \\
0 \\
0\end{array}$ & $\mid \begin{array}{l}0 \\
\stackrel{0}{1} \\
0 \\
0\end{array}$ & $\frac{0}{\circ}$ & $\frac{\circ}{5}$ & $\begin{array}{l}\vdots \\
\text { ô } \\
\text { in }\end{array}$ & $\begin{array}{l}0 \\
\infty \\
\stackrel{0}{\infty} \\
\end{array}$ & $\begin{array}{l}\stackrel{0}{m} \\
\stackrel{m}{m}\end{array}$ & $\begin{array}{l}0 \\
8 \\
0 \\
0 \\
0 \\
10\end{array}$ & 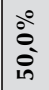 & $\begin{array}{l}\circ \\
\vdots \\
\circ \\
\text { in }\end{array}$ & $\begin{array}{l}0 \\
0 \\
0 \\
0\end{array}$ & \begin{tabular}{|l|}
0 \\
0 \\
$\circ$ \\
ก
\end{tabular} & $\begin{array}{l}\stackrel{0}{0} \\
\dot{0} \\
\stackrel{0}{0}\end{array}$ & $\stackrel{\circ}{\circ}$ & $\begin{array}{l}\text { ¿̊ } \\
\text { iे } \\
\text { î }\end{array}$ \\
\hline & 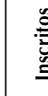 & สิ & $\sigma$ & i & $\checkmark 0$ & & $\sim$ & - & สิ & 0 & $\wedge$ & $n$ & $\stackrel{2}{2}$ & $a$ & 0 & + & 0 & N & 0 & $\sigma$ & - & - & هి \\
\hline & 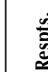 & $\sigma$ & $m$ & 0 & \begin{tabular}{l|l}
$\sigma$ & $\nabla$
\end{tabular} & & - & - & $\cong$ & + & 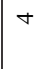 & t & $=$ & $n$ & N & $\sim$ & $m$ & - & 0 & $\sim$ & 0 & 0 & $\stackrel{L}{m}$ \\
\hline \multirow{3}{*}{ 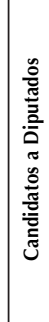 } & $\circ$ & $\begin{array}{l}\stackrel{\circ}{~} \\
\text { ஸे }\end{array}$ & $\begin{array}{l}\stackrel{\circ}{+} \\
\stackrel{\circ}{\circ}\end{array}$ & $\frac{0}{2}$ & 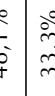 & 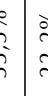 & 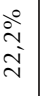 & $\begin{array}{l}\stackrel{0}{0}_{0} \\
\infty^{-} \\
\stackrel{0}{0}\end{array}$ & 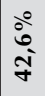 & $\begin{array}{l}\stackrel{0}{\circ} \\
\stackrel{n}{7}\end{array}$ & $\begin{array}{l}\stackrel{0}{\circ} \\
i \\
\stackrel{0}{+}\end{array}$ & $\begin{array}{l}\circ \\
\stackrel{0}{0} \\
\stackrel{0}{\circ}\end{array}$ & $\begin{array}{l}\dot{0} \\
m \\
m \\
m\end{array}$ & 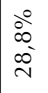 & $\begin{array}{l}\delta^{0} \\
\infty^{\circ} \\
\tilde{m}^{\prime}\end{array}$ & 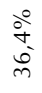 & $\begin{array}{l}\stackrel{0}{2} \\
i p \\
\text { in }\end{array}$ & $\frac{\circ}{\frac{0}{n}}$ & $\begin{array}{l}\stackrel{0}{m} \\
\text { mे } \\
\text { mे }\end{array}$ & $\mid \begin{array}{c}\stackrel{0}{0} \\
i n \\
\infty \\
m\end{array}$ & $\begin{array}{l}\stackrel{0}{0} \\
\stackrel{0}{0} \\
\text { in }\end{array}$ & $\begin{array}{l}\text { o̊ } \\
\text { ¿े } \\
\text { in }\end{array}$ & ì \\
\hline & 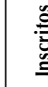 & సิ & เ & 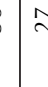 & $\bar{v} \bar{\gamma}$ & & $a$ & $n$ & $\stackrel{\stackrel{20}{=}}{=}$ & ชె & $m$ & ঃ & ָิ & or & in & $=$ & $\ddot{\sim}$ & $\wedge$ & $m$ & $m$ & $\infty$ & $\infty$ & 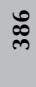 \\
\hline & $\begin{array}{ll}0 \\
.\end{array}$ & $\mp$ & 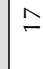 & $: 7$ & $c 1$ & & $\sim$ & $N$ & $q$ & $\hat{\imath}$ & $\stackrel{0}{2}$ & 0 & \& & 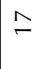 & $\because$ & + & 은 & $\theta$ & - & in & $\sigma$ & 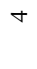 & 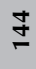 \\
\hline & & 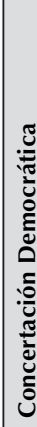 & 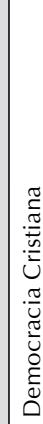 & 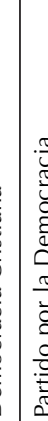 & 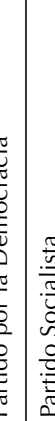 & 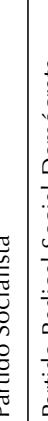 & 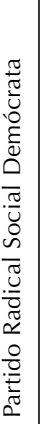 & 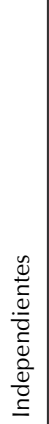 & 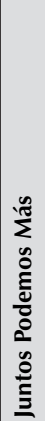 & 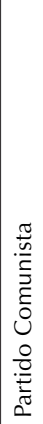 & 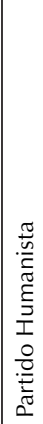 & 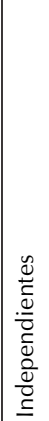 & 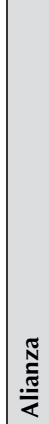 & 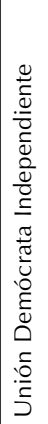 & 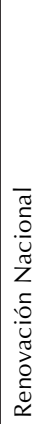 & 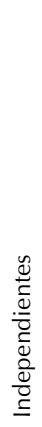 & 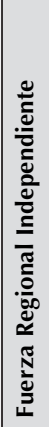 & 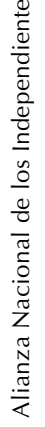 & 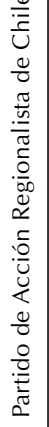 & 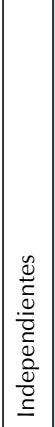 & 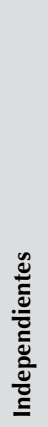 & 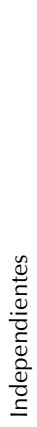 & 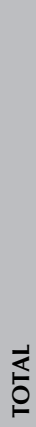 \\
\hline
\end{tabular}




\section{DIPUTADOS ELECTOS QUE NO RESPONDIERON}

\begin{tabular}{|c|c|c|c|c|c|}
\hline $\mathrm{N}^{0}$ & Reg. & Dist. & Diputado(a) & Pacto & Partido \\
\hline 1 & 1 & 1 & Paredes Fierro, Iván & Concertación & PS \\
\hline 2 & 1 & 1 & Valcarce Becerra, Ximena & Alianza & $\mathrm{RN}$ \\
\hline 3 & I & 2 & Rossi Ciocca, Fulvio & Concertación & PS \\
\hline 4 & II & 3 & Espinosa Monardes, Marcos & Concertación & PRSD \\
\hline 5 & II & 4 & Araya Guerrero, Pedro & Concertación & DC \\
\hline 6 & II & 4 & Rojas Molina, Manuel & Alianza & UDI \\
\hline 7 & III & 5 & Leal Labrin, Antonio & Concertación & PPD \\
\hline 8 & III & 5 & Aedo Ormeño, René & Alianza & RN \\
\hline 9 & III & 6 & Mulet Martínez, Jaime & Concertación & DC \\
\hline 10 & III & 6 & Robles Pantoja, Alberto & Concertación & PRSD \\
\hline 11 & IV & 7 & Bertolino Rendic, Mario & Alianza & RN \\
\hline 12 & IV & 8 & Encina Moriamez, Francisco & Concertación & PS \\
\hline 13 & IV & 9 & Fuentealba Vildósola, Renán & Concertación & DC \\
\hline 14 & V & 11 & Núñez Lozano, Marco Antonio & Concertación & PPD \\
\hline 15 & V & 12 & Bustos Ramírez, Juan & Concertación & PS \\
\hline 16 & V & 12 & Herrera Silva, Amelia & Alianza & RN \\
\hline 17 & V & 13 & Soto González, Laura & Concertación & PPD \\
\hline 18 & V & 13 & Godoy Ibáñez, Joaquín & Alianza & RN \\
\hline 19 & V & 14 & Chahuan Chahuan, Francisco & Alianza & RN \\
\hline 20 & V & 15 & Venegas Rubio, Samuel & Concertación & PRSD \\
\hline 21 & V & 15 & Eluchans Urenda, Edmundo & Alianza & UDI \\
\hline 22 & RM & 16 & Silber Romo, Gabriel & Concertación & DC \\
\hline 23 & RM & 18 & Olivares Zepeda, Carlos & Concertación & DC \\
\hline 24 & RM & 18 & Girardi Briere, Guido & Concertación & PPD \\
\hline 25 & RM & 19 & Nogueira Fernández, Claudia & Alianza & UDI \\
\hline 26 & RM & 22 & Cardemil Herrera, Alberto & Alianza & Ind \\
\hline 27 & RM & 25 & Salaberry Soto, Felipe & Alianza & UDI \\
\hline \multirow[t]{2}{*}{28} & RM & 28 & Inzunza Gregorio De & & \\
\hline & & & Las Heras, Jorge & Concertación & PPD \\
\hline 29 & RM & 29 & Allende Bussi, Isabel & Concertación & PS \\
\hline 30 & RM & 29 & Errázuriz Eguiguren, Maximiano & Alianza & RN \\
\hline 31 & RM & 30 & Kast Rist, José Antonio & Alianza & UDI \\
\hline 32 & VI & 33 & Sule Fernández, Alejandro & Concertación & PRSD \\
\hline 33 & VI & 33 & Bauer Jouanne, Eugenio & Alianza & UDI \\
\hline 34 & VI & 34 & Sepúlveda Orbenes, Alejandra & Concertación & DC \\
\hline 35 & VI & 34 & Masferrer Pellizzari, Juan & Alianza & UDI \\
\hline 36 & VI & 35 & Latorre Carmona, Juan Carlos & Concertación & DC \\
\hline 37 & VI & 35 & Barros Montero, Ramón & Alianza & UDI \\
\hline 38 & VII & 36 & León Ramírez, Roberto & Concertación & DC \\
\hline 39 & VII & 37 & Aguilo Melo, Sergio & Concertación & PS \\
\hline 40 & VII & 38 & Lorenzini Basso, Pablo & Concertación & DC \\
\hline \multirow[t]{2}{*}{41} & VII & 38 & Álvarez-Salamanca & & \\
\hline & & & Büchi, Pedro Pablo & Alianza & RN \\
\hline
\end{tabular}




\begin{tabular}{|c|c|c|c|c|c|}
\hline $\mathbf{N}^{\circ}$ & Reg. & Dist. & Diputado(a) & Pacto & Partido \\
\hline 42 & VII & 39 & Tarud Decarret, Jorge & Concertación & PPD \\
\hline 43 & VII & 39 & Palma Flores, Osvaldo & Alianza & $\mathrm{RN}$ \\
\hline 44 & VII & 40 & Ceroni Fuentes, Guillermo & Concertación & PPD \\
\hline 45 & VII & 40 & Urrutia Bonilla, Ignacio & Alianza & UDI \\
\hline 46 & VIII & 41 & Martínez Labbé, Rosauro & Alianza & $\mathrm{RN}$ \\
\hline 47 & VIII & 42 & Sabag Villalobos, Jorge & Concertación & DC \\
\hline 48 & VIII & 42 & Monckeberg Díaz, Nicolás & Alianza & $\mathrm{RN}$ \\
\hline 49 & VIII & 43 & Sunico Galdames, Raúl & Concertación & DC \\
\hline 50 & VIII & 43 & Ulloa Aguillon, Jorge & Alianza & UDI \\
\hline 51 & VIII & 44 & Ortiz Novoa, José Miguel & Concertación & DC \\
\hline 52 & VIII & 44 & Egaña Respaldiza, Andrés & Alianza & UDI \\
\hline 53 & VIII & 45 & Pacheco Rivas, Clemira & Concertación & PS \\
\hline 54 & VIII & 45 & Bobadilla Muñoz, Sergio & Alianza & UDI \\
\hline 55 & VIII & 46 & Monsalve Benavides, Manuel & Concertación & PS \\
\hline 56 & VIII & 46 & Norambuena Farías, Iván & Alianza & UDI \\
\hline 57 & VIII & 47 & Pérez Arriagada, José & Concertación & PRSD \\
\hline 58 & IX & 48 & Venegas Cárdenas, Mario & Concertación & DC \\
\hline 59 & IX & 48 & Arenas Hodar, Gonzalo & Alianza & UDI \\
\hline 60 & IX & 49 & Quintana Leal, Jaime & Concertación & PPD \\
\hline 61 & IX & 49 & Estay Peñaloza, Enrique & Alianza & UDI \\
\hline 62 & IX & 50 & Saffirio Suárez, Eduardo & Concertación & DC \\
\hline 63 & IX & 50 & Becker Alvear, Germán & Alianza & $\mathrm{RN}$ \\
\hline 64 & IX & 51 & Díaz Del Río, Eduardo & Concertación & Ind \\
\hline 65 & IX & 52 & Meza Moncada, Fernando & Concertación & PRSD \\
\hline 66 & IX & 52 & García García, René Manuel & Alianza & $\mathrm{RN}$ \\
\hline 67 & $x$ & 53 & De Urresti Longton, Alfonso & Concertación & PS \\
\hline 68 & $x$ & 53 & Delmastro Naso, Roberto & Alianza & Ind \\
\hline 69 & $x$ & 54 & Jaramillo Becker, Enrique & Concertación & DC \\
\hline \multirow[t]{2}{*}{70} & $x$ & 54 & Von Mühlenbrock Zamora, & & \\
\hline & & & & Alianza & UDI \\
\hline 71 & $x$ & 55 & Ojeda Uribe, Sergio & Concertación & DC \\
\hline 72 & $x$ & 55 & Hernández Hernández, Javier & Alianza & UDI \\
\hline 73 & $x$ & 56 & Espinoza Sandoval, Fidel & Concertación & PS \\
\hline 74 & $x$ & 57 & Turres Figueroa, Marisol & Alianza & UDI \\
\hline 75 & $x$ & 58 & Ascencio Mansilla, Gabriel & Concertación & DC \\
\hline 76 & $x$ & 58 & Alvarado Andrade, Claudio & Alianza & UDI \\
\hline 77 & $X I$ & 59 & Alinco Bustos, René & Concertación & PPD \\
\hline 78 & $X I$ & 59 & Galilea Carrillo, Pablo & Alianza & $\mathrm{RN}$ \\
\hline 79 & XII & 60 & Goic Boroevic, Carolina & Concertación & DC \\
\hline 80 & XII & 60 & Álvarez Zenteno, Rodrigo & Alianza & UDI \\
\hline
\end{tabular}




\section{DIPUTADOS ELECTOS \\ QUE SÍ RESPONDIERON}

\begin{tabular}{|c|c|c|c|c|c|}
\hline$N^{\circ}$ & Reg. & Dist. & Diputado(a) & Pacto & Partido \\
\hline 1 & 1 & 2 & Isasi Barbieri, Marta Eliana & FRI & PAR \\
\hline 2 & II & 3 & Ward Edwards, Felipe & Alianza & UDI \\
\hline 3 & IV & 7 & Díaz Díaz, Marcelo & Concertación & PS \\
\hline 4 & IV & 8 & Walker Prieto, Patricio & Concertación & DC \\
\hline 5 & IV & 9 & Muñoz D’Albora, Adriana & Concertación & PPD \\
\hline 6 & $\mathrm{~V}$ & $\begin{array}{r}10 \\
\operatorname{arco}\end{array}$ & $\begin{array}{l}\text { Enríquez-Ominami Gumucio, } \\
\text { Concertación }\end{array}$ & PS & \\
\hline 7 & V & 10 & Vargas Lyng, Alfonso & Alianza & $\mathrm{RN}$ \\
\hline 8 & V & 11 & Forni Lobos, Marcelo & Alianza & UDI \\
\hline 9 & V & 14 & González Torres, Rodrigo & Concertación & PPD \\
\hline 10 & RM & 16 & Melero Abaroa, Patricio & Alianza & UDI \\
\hline 11 & RM & 17 & Saa Díaz, María Antonieta & Concertación & PPD \\
\hline 12 & RM & 17 & Rubilar Barahona, Karla & Alianza & $\mathrm{RN}$ \\
\hline 13 & RM & 19 & Hales Dib, Patricio & Concertación & PPD \\
\hline 14 & RM & 20 & Escobar Ruffatt, Alvaro & Concertación & PPD \\
\hline 15 & RM & 20 & Sepúlveda Hermosilla, Roberto & Alianza & RN \\
\hline 16 & RM & 21 & Burgos Varela, Jorge & Concertación & DC \\
\hline 17 & RM & 21 & Cubillos Sigall, Marcela & Alianza & UDI \\
\hline 18 & RM & 22 & Tohá Morales, Carolina & Concertación & PPD \\
\hline 19 & RM & 23 & Monckeberg Bruner, Cristián & Alianza & RN \\
\hline 20 & RM & 23 & Dittborn Cordua, Julio & Alianza & UDI \\
\hline 21 & RM & 24 & Accorsi Opazo, Enrique & Concertación & PPD \\
\hline 22 & RM & 24 & Cristi Marfil, María Angélica & Alianza & UDI \\
\hline 23 & RM & 25 & Vidal Lázaro, Ximena & Concertación & PPD \\
\hline 24 & RM & 26 & Duarte Leiva, Gonzalo & Concertación & DC \\
\hline 25 & RM & 26 & Montes Cisternas, Carlos & Concertación & PS \\
\hline 26 & RM & 27 & Jiménez Fuentes, Tucapel & Concertación & Ind \\
\hline 27 & RM & 27 & Moreira Barros, Iván & Alianza & UDI \\
\hline 28 & RM & 28 & Paya Mira, Darío & Alianza & UDI \\
\hline 29 & RM & 30 & Farías Ponce, Ramón & Concertación & PPD \\
\hline 30 & $\mathrm{RM}$ & 31 & Pascal Allende, Denise & Concertación & PS \\
\hline 31 & RM & 31 & Uriarte Herrera, Gonzalo & Alianza & UDI \\
\hline 32 & VI & 32 & Valenzuela Van Treek, Esteban & Concertación & PPD \\
\hline 33 & VI & 32 & $\begin{array}{l}\text { García Huidobro Sanfuentes, } \\
\text { Alejandro }\end{array}$ & Alianza & UDI \\
\hline 34 & VII & 36 & Correa De La Cerda, Sergio & Alianza & UDI \\
\hline 35 & VII & 37 & Verdugo Soto, Germán & Alianza & $\mathrm{RN}$ \\
\hline 36 & VIII & 41 & Jarpa Wevar, Carlos Abel & Concertación & PRSD \\
\hline 37 & VIII & 47 & Lobos Krause, Juan & Alianza & UDI \\
\hline 38 & IX & 51 & Tuma Zedan, Eugenio & Concertación & PPD \\
\hline 39 & $x$ & 56 & Recondo Lavanderos, Carlos & Alianza & UDI \\
\hline 40 & $x$ & 57 & Vallespin López, Patricio & Concertación & DC \\
\hline
\end{tabular}


SENADORES ELECTOS QUE SÍ RESPONDIERON

\begin{tabular}{|cll|}
\hline Región & CIRCUNSCRIPCIÓN & NOMBRE \\
\hline II & Segunda & Carlos Cantero Ojeda \\
IV & Cuarta & Evelyn Matthei Fornet \\
RM & Séptima & Jovino Novoa Vásquez \\
RM & Octava & Juan Pablo Longueira Montes \\
RM & Octava & María Soledad Alvear Valenzuela \\
VI & Novena & Andrés Pío B. Chadwick Piñera \\
VIII & Décimo segunda & Hosaín Sabag Castillo \\
VIII & Décimo tercera & Víctor Pérez Varela \\
X & Décimo séptima & Camilo Escalona Medina \\
XII & Décimo novena & Pedro Muñoz Aburto \\
\hline
\end{tabular}

SENADORES ELECTOS QUE NO RESPONDIERON

\begin{tabular}{|cll|}
\hline Región & CIRCUNSCRIPCIÓN & NOMBRE \\
\hline II & Segunda & José Antonio Gómez Urrutia \\
IV & Cuarta & Jorge Pizarro Soto \\
RM & Septima & Guido Girardi Lavín \\
VI & Novena & Juan Pablo Letelier Morel \\
VIII & Décimo segunda & Alejandro Navarro Brain \\
VIII & Décimo tercera & Mariano Ruíz-Esquide Jara \\
X & Décimo sexta & Andres Allamand Zavala \\
X & Décimo sexta & Eduardo Frei Ruiz-Tagle \\
X & Décimo séptima & Carlos Ignacio Kuschel Silva \\
XII & Décimo novena & Carlos Antonio Karin Bianchi Chelech \\
\hline
\end{tabular}


LISTA DE SENADORES QUE NO SE REELEGÍAN

QUE SÍ CONTESTARON

\begin{tabular}{|cll|}
\hline Región & CIRCUNSCRIPCIÓN & NOMBRE \\
\hline I & Primera & Jaime Orpis \\
III & Tercera & Baldo Procurica \\
V & Quinta & Carlos Ominami \\
V & Quinta & Sergio Romero \\
V & Sexta & Nelson Ávila \\
V & Sexta & Jorge Arancibia \\
VII & Décima & Juan Antonio Coloma \\
VII & Décimo primera & Hernán Larraín \\
XI & Décimo octava & Antonio Horvarth \\
\hline
\end{tabular}

LISTA DE SENADORES QUE NO SE REELEGÍAN QUE NO CONTESTARON

\begin{tabular}{|cll|}
\hline Región & CIRCUNSCRIPCIÓN & NOMBRE \\
\hline I & Primera & Fernando Flores \\
III & Tercera & Ricardo Nuñez \\
VII & Décima & Jaime Gazmuri \\
VII & Décimo primera & Jaime Naranjo \\
IX & Décimo cuarta & Alberto Espina \\
IX & Décimo cuarta & Roberto Muñoz \\
IX & Décimo quinta & Guillermo Vázquez \\
IX & Décimo quinta & José García \\
XI & Décimo octava & Adolfo Zaldívar \\
\hline
\end{tabular}

